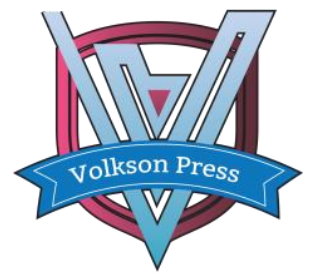

Contents List available at VOLKSON PRESS

Economics \& Management Innovations(EMI)

DOI : http://doi.org/10.26480/icemi.01.2017.328.330

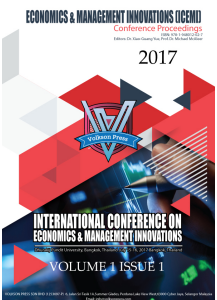

\title{
Comparative Study of Using Word Co-occurrence to Determine Symptoms from Texts
}

\author{
Chaveevan Pechsiri, ${ }^{1, *}$ \\ College of Innovative of Tech.\&Engineering, Dhurakij Pundit University, Thailand \\ *Chaveevan.pec@DPU.ac.th
}

This is an open access article distributed under the Creative Commons Attribution License, which permits unrestricted use, distribution, and reproduction in any medium, provided the original work is properly cited.

\section{ARTICLE DETAILS}

\section{Article History:}

\section{Received 02 october 2017}

Accepted 06 october 2017

Available online 11 october 2017

\section{Keywords:}

word co-occurrence, event

boundary, Elementary Discourse Unit.

\section{ABSTRACT}

The research approach is to determine the symptom explanation from downloaded hospital documents to construct the semantic relations between disease-topic names and symptom explanation for enhancing the automatic problem-solving system. However, this paper aims to comparative study of using different word co-occurrence sizes as the two word co-occurrence and the $\mathrm{N}$ word co-occurrence on verb phrases to identify the symptomconcept sentences. We also use these word co-occurrences to determine the boundary of the consecutivesymptomconcept sentences through the machine learning technique and the similarity-score determination. The research results provide the high precision of the symptom determination from the documents.

\section{Introduction}

The research objective is the comparative study of using word cooccurrence to determine the explanation of disease-symptom-concept sentences from the downloaded health-care documents on the hospital web-boards. The research results are beneficial to the automatic diagnosis system. Moreover, the disease symptom explanation mostly consists of event expressions on several EDUs (where EDU is an Elementary Discourse Unit expression defined as a simple sentence or a clause, [1]) as the symptom-concept explanation on a document of a certain disease as follow.

Example1.

Topicname:โรคกระเพาะ/StomachDisease

EDU1symptom: "[หนู]ปวดทองอย?างหนัก" / "[หนู / A user] ปวด/pain ทีอง /abdominal อย团งหนัก/heavily" ([A user] has a stomachache heavily.)

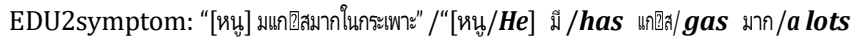

([He] has lots of gas in the stomach.)

ในกระเพาะ/inside stomach"

EDU3symptom: "อาการมักเปนหลงอาหารเย็น"/“อาการ/Symptom มักเปนเ/mostly occurs หลั้งอาหารเยน็ /after dinner" (The symptom mostly occurs after dinner.)

EDU4: "[หนู] สงสัยเปนโรคกระเพาะ" ([He] doubts to get gastropathy.

where the [..] symbol means ellipsis. A symptom-concept EDU boundary occurs on EDU1, EDU2, and EDU3. According to Example1, the research emphasizes on the event expressions by verb phrases because of most symptom-concept expressions on the verb phrases of EDUs. Each verb phrase is based on the following linguistic pattern after stemming words and eliminating stop words.

EDU ? NP1 VP

VP ? V1 |V1 NP2 |V1 Adverb |V2 NP3 |V2 NP3 VP| V2 Adj V1

? $V_{\text {strong }}$ Preverb $V_{\text {strong }}$

V2 ? V weak | Preverb Vweak

NP1? Noun1 | Noun2 | Noun3

NP2 ? Noun2 | Noun2 NP2 | Noun2 AdjectivePhrase NP3? Noun3 |

Noun3 Adj prep NP2
Noun1? \{'ผูไthย/patient' 'โรค/disease'...\}

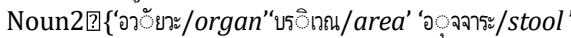

...\} Noun3? \{'อาการ/symptom' 'แผล/scar' 'รอย/mark'

'ไข|/fever' 'ผื่น/rash' 'หนอง/pus' ...\}

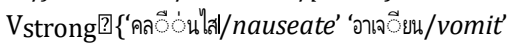

'ปวด/pain' 'เจ็บ/pain' "แนน/constrict' 'คัน/itchy'...\}

Vweak ? \{'เป็/be' 'มี/have'รณึึก/feel'\}

Adv? $\{$ 'ยาก/difficultly' .... $\}$; Adj ใ ? \{'สอี.../...color' 'เหลว/watery' .... ; Preverb ? $\{$ 'ไม่/not' ... $\}$

where NP1, NP2, and NP3 are noun phrases. VP is a verb phrase. Vstrong is a strong verb set with the symptom concept. $V_{\text {weak }}$ is a weak verb set which need more information to have the symptom concept. Noun3 is a noun set with a symptom concept. Adv is an adverb set with the symptom concept. Adj is the adjective set with the symptom concept. prep is a preposition.

Moreover, previous researches have worked on event extraction from text [2, 3, 4]. In 2011, [2] applied syntactic and lexical constraints on binary relations expressed by verb phrases (called relation phrases) for the Open Information Extraction system, REVERB. They implemented a verb-noun combination on the relation phrase to match the POS tag pattern. The research results with more than $30 \%$ of REVERB's extractions are at precision 0.8. Whereas [3] proposed methods for filtering harmful sentences based on multiple word co-occurrences. They compare harmless rate between two-word co-occurrence and threeword co-occurrence. The precision of identify and filtering the harmful sentences through three-word co-occurrence method exceeds 90\% whereas the precision of the two-word co-occurrence is under $50 \%$. In 2014 , [4] worked on a model for identifying causality in verb-noun pairs to encode cause or non-cause relation. The result of this research achieves $14.74 \%$ and $41.9 \%$ F-scores for the basic supervised classifier. Thus, most of previous researches identify an event by two-word cooccurrence as verb-noun co-occurrence. Whereas the symptom-concept expression on each EDU of our research mostly consists of several words (see Example 1). However, our research contains two problems of determining the explanation of the symptom-concept EDUs; how to identify an EDU verb phrase having symptom concept (see Example1) and how to determine the symptom explanation as the symptom-concept 
EDU boundary, i.e. EDU1-EDU3 of the Example1. Therefore, we propose using the different word co-occurrence sizes; the co-occurrence between two words (called Word-Co) and the co-occurrence between $\mathrm{N}$ words (called N-Word-Co), on the EDU verb phrase for comparative study of using Word-Co and N-Word-Co to determine the symptom-concept EDU. With regard to the symptom-concept EDU boundary determination, we apply Support Vector Machine (SVM) [5] to solve the boundary having Word-Co as input features and we apply the similarity score [6] to solve the boundary having N-Word-Co as input features. Moreover, we also determine the $\mathrm{N}$-Word-Co size (or the $\mathrm{N}$ value) by SVM.

The research consists of 3 sections. In section 2, the research framework of determining the symptom EDU vector is described and we evaluate and conclude our proposed model in section3.

\section{A Framework of Determining Symptom Vector}

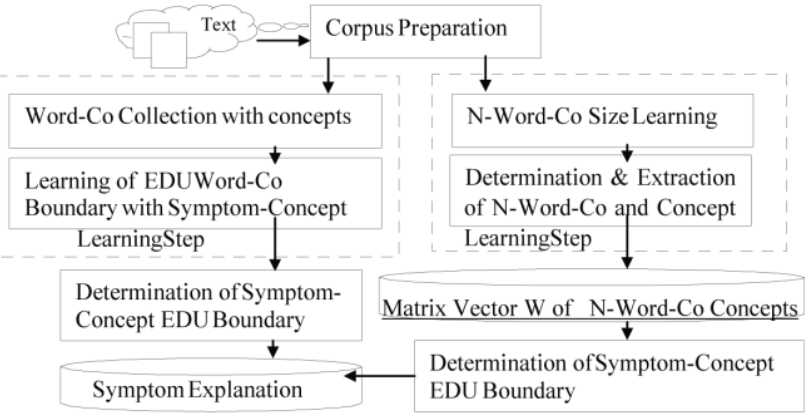

Figure1. System Overview

There are three main steps in determining the symptom vector or the symptom-concept EDU vector by using the Word-Co or N-Word-Co technique, Corpus Preparation step, Learning Step and Symptom Vector Determination Step.

\subsection{Corpus Preparation}

This step is the preparation of corpus in the form of EDU from the medical-care documents on the hospital's web-board (http://haamor.com/). The step involves with using Thai word segmentation tools [7] including Name entity [8] followed by EDU segmentation [9]. These annotated EDUs are used as an EDU corpus which contains 3000 EDUs of gastrointestinal tract diseases and childhood diseases and is separated into 2 parts; the first part of 2000EDUs for the learning step of both Word-Co and N-Word-Co; and the second part of 1000 EDUs for determining the symptom-concept EDU boundary. We then semi-automatically annotate the Word-Co expressions with symptom concepts for the $\mathrm{w} 1$ and w2 tags as Word-Co and for the w1 through wi as $\mathrm{N}$-Word-Co after stemming words and the stop word removal as shown in Fig.2. All symptom concepts are referred to WordNet (http://word-net.princeton.edu/obtain) and MeSH (https://www. nlm.nih.gov/mesh/) after translating from Thai to English, by Lexitron (http://lexitron.nectec.or.th/).

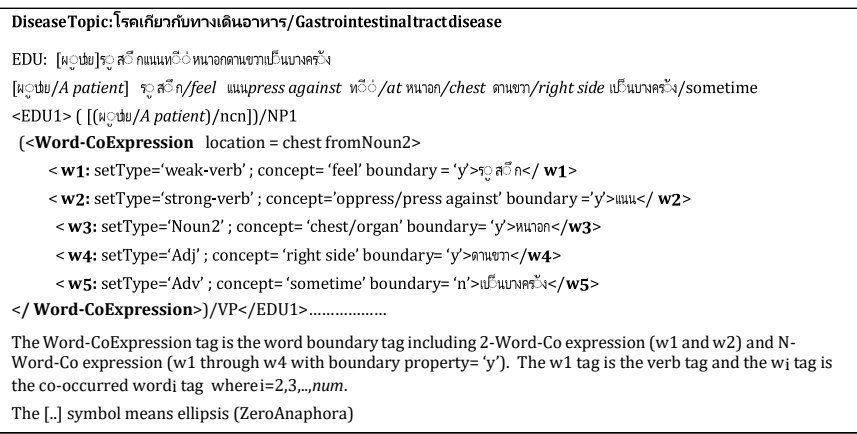

Figure2. Word Co-Occurrence Annotation

\section{$2.2 \quad$ Learning}

2.2.1 Word-Co Learning. We collect each Word-Co feature, w1w2 or $v_{C o} w_{C O}$, with the symptom concept into VW from annotated corpus where VW is a Word-Co set with the symptom concepts; $w 1$ is a verb represented by $v_{c o}$; and $w 2$ is a co-occurred word represented by $w_{c o}$. VW is used for identifying and extracting the consecutive symptomconcept Word-Co occurrences for learning the EDU's Word-Co boundary with the symptom concept by SVM (using Weka, ,http://www.cs. wakato.ac.nz/ml/weka/). SVM is the linear kernel: the linear function, $f(\mathrm{x})$, of the input $\mathrm{x}=(\mathrm{x} 1 \ldots \mathrm{xn})$

assigned to the positive class if $f(x) \geq 0$, and otherwise to the negative class if $f(x)<0$, can be written as

$$
\begin{aligned}
f(\mathrm{x}) & =\langle\underset{n}{\mathrm{wt}} \cdot \mathrm{x}\rangle+b \\
& =\sum_{j=1} w t_{j} x_{j}+b
\end{aligned}
$$

where $\mathrm{x}$ is a dichotomous vector number, wt is a weight vector, $b$ is a bias, and $(\mathrm{w}, b) ? \mathrm{R}{ }^{n}$ ? $\mathrm{R}$ are the parameters that control the function. The SVM learning is to determine $w t j$ and $b$ for each Word-Co feature, $v c o-j w c o-$ $j(x j)$ in each Word-Co pair, $v$ co-j $w c o-j v c o-j+1 w c o-j+1$, from the supervised learning of SVM by sliding the window size of two consecutive EDUs with one sliding EDU distance where $j=1,2, \ldots, n$ and $n$ is End-of-Boundary.

2.2.2. N-Word-Co Learning. In regard to Eq. 2, the features used for learning N-Word-Co size by SVM are obtained by the following concept sets: Verbstrong, Verb weak, Noun1, Noun2, Noun3,Adj, and Adv. The SVM learning is to determine $w t_{j}$ and $b$ for each word feature, $w_{j}$ (or $x_{j}$ ) in each word-concept pair $(w j w j+1$ ) with a symptom concept. The N-Word-Co size/boundary learning from $w j w j+1$ of VP is the supervised learning of SVM by sliding the window size of two consecutive words with one sliding word distance after stemming words and the stop word removal. Where $j=1,2, . ., n$ and $n$ is End-of-Boundary and is equivalent to the $\mathrm{N}$ value of $\mathrm{N}$ Word-Co size.

\subsection{Symptom-Concept EDU Boundary Determination}

2.3.1 Symptom-Concept EDU Boundary Determination by Using Word-Co. After using VW to identify a symptom concept EDU from the testing corpus, the wt vector of all $v c o-j w c o-j$ from the SVM learning are used to determine the boundary of the symptom-concept EDUs with Eq. 1 by sliding the window size of two consecutive EDUs with one sliding EDU distance. If $f(\mathrm{x})<0$ then the boundary is ended as a symptom vector; otherwise continuing.

Symptom-Concept EDU Boundary Determination by Using N-Word-Co. The symptom- concept EDU boundary is determined after the N-Word-Co size determination and extraction. After $w 1$ ? $\mathrm{V}_{\text {strong? }} \mathrm{V}_{\text {weak }}$ and $w 1$ is the first word of VP on the testing corpus, the wt vector of all $w j$ from the SVM learning in section 2.2.2 which are used to determine and extract the $\mathrm{N}$-WordCo size/boundary with symptom-concept into the matrix vector $\mathrm{W}$ of symptom concepts with Eq. 1 by sliding the window size of two consecutive words with one sliding word distance. If $f(\mathrm{x})<0$ then the boundary is ended as a word vector of N-Word-Co; otherwise continuing. The symptom-concept EDU boundary is then determined by the similarity score determination as Max Similarity Score (MaxSimScore) [6] in Eq. 2. MaxSimScore is determined between the N-Word-Co of the testing corpus's EDU and the candidate N-Word-Co expressions from $\mathrm{W}$. The N-Word-Co concept of each consecutive EDU verb phrase is the symptom concept if MaxSimScore $\geq 0.9$ to $\mathrm{W}$; otherwise the symptom vector is ended.

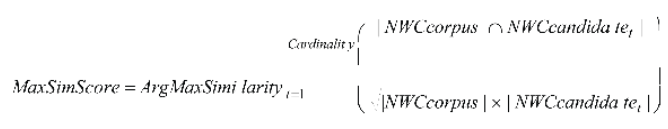

where Cardinality is the number of $\mathrm{N}$ - Word - Co elements of $\mathrm{W}$

$\mathrm{W}$ is the Matrix vector of $\mathrm{N}$ - Word - Co (the $\mathrm{N}$ - Word - Co set) with the symptom concept

NWCcandida te is a candidate $\mathrm{N}$ - Word - Co element of The the N - Word - Co set with the symptom concept

NWCcorpus is an $\mathrm{N}$ - Word - Co of EDU from the testing corpus 


\section{Evaluation and Conclusions}

The testing corpus of 1000 EDUs of gastrointestinal tract diseases and childhood diseases is used for evaluating the symptom-concept-EDUboundary determination. Both evaluations of the symptom vector determinations by using Word-Co and by using N-Word-Co from the testing corpus are based on the precision and the recall which are evaluated by three expert judgments with max win voting. The precisions of determining the symptom-concept EDU boundaries are $91.3 \%$ and $84.8 \%$ with the recalls of $69.2 \%$ and $73.1 \%$ by using N-WordCo and Word-Co respectively. The reason of low recall is the anaphora problem, i.e. '(บางสิ่ง/something)/pronoun' occurrence on VP as follow.

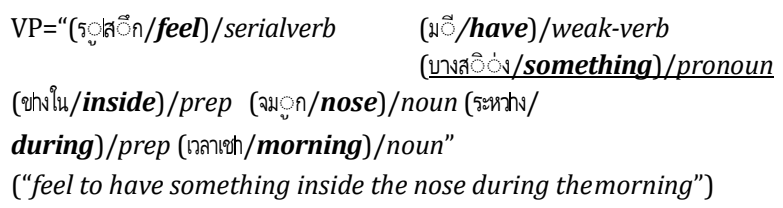

However, the research results provide the higher precision by using NWord-Co and the higher recall by using Word-Co to determine the symptom explanation from the documents. Thus, the symptom explanation is determined to construct the semantic relation as the diseaseName- symptomExplanation relation where the disease-names are obtained by the document topics. The relation is beneficial to the automatic diagnosis of the problem solution. Moreover, the proposed method of using word co-occurrence to determine the symptom explanation can also be applied to the other areas such as the industrial finance problem explanation.

\section{Acknowledgement}

This work has been supported by JEDI, Dhurakij Pundit University.

\section{References}

[1] L. Carlson, D.Marcu, and M. E. Okurowski: Building a DiscourseTagged Corpus in the Framework of Rhetorical Structure Theory. In Current Directions in Discourse and Dialogue. (2003), p.85-112

[2] A.Fader, S. Soderland, and O.Etzioni: Identifying Relations for Open Information Extraction. In Proc. of the Conference on Empirical Methods in Natural Language Processing,(2011), p.1535-1425.

[3] S.Ando,Y. Fujii, and T. Ito: Filtering Harmful Sentences based on Multiple Word Co-occurrence. IEEE/ACIS 9th International Conference on Computer and Information Science(ICIS), (2010).

[4] M.Riaz and R. Girju:Recognizing Causality in Verb- Noun Pairs via Noun and Verb Semantics. In Proc. of the EACL 2014 Workshop on Computational Approaches to Causality in Language, (2014), p.48-57.

[5] T.M.Mitchell: Machine Learning. The McGraw-Hill Companies Inc. and MIT Press, Singapore (1997).

[6] S.Biggins, S.Mohammed, S.Oakley: University Of Sheffield: Two Approaches to Semantic Text

Similarity. In Proceedings of First Joint Conference on Lexical and Computational Semantics, Montre'al, Canada,(2012), p.655-661.

[7] S. Sudprasert and A. Kawtrakul: Thai Word segmentation based on Global and Local Unsupervised Learning. In Proceedings of the 7th National Computer Science and Engineering Conference (2003).

[8] H.Chanlekha and A. Kawtrakul:Thai Named Entity Extraction by incorporating Maximum Entropy Model with Simple Heuristic Information. First International Joint Conference,Hainan Island,China, (2004).

[9] J .Chareonsuk, T. Sukvakree, and A. Kawtrakul: Elementary Discourse unit Segmentation for Thai using Discourse Cue and Syntactic Information. In Proceedings of the 9th National Computer Science and Engineering Conference (2005). 